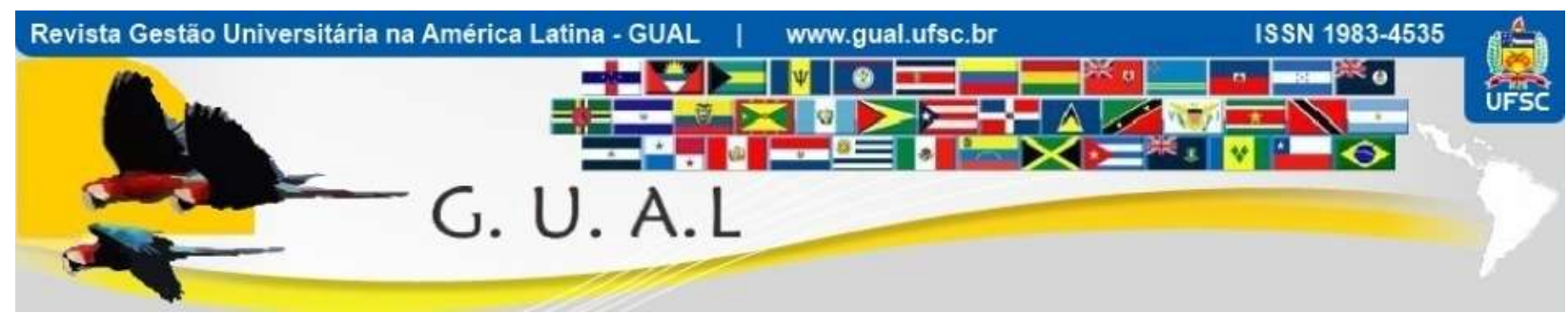

DOI: http://dx.doi.org/10.5007/1983-4535.2020v13n2p25

\title{
GESTÃO DE PROJETOS NO CONTEXTO DA APRENDIZAGEM BASEADA EM PROJETOS USANDO O MODELO LIFE CYCLE CANVAS®
}

\section{PROJECT MANAGEMENT IN THE CONTEXT OF PROJECT-BASED LEARNING USING THE MODEL LIFE CYCLE CANVAS $®$}

\author{
Thayse Hanne Câmara Ribeiro do Nascimento, Mestre \\ https://orcid.org/0000-0002-1799-0798 \\ thaysehanne@gmail.com \\ Universidade Federal do Rio Grande do Norte | Faculdade de Ciências da Saúde do Trairi \\ Santa Cruz | Rio Grande do Norte | Brasil \\ Dmitryev Cyreneu da Silva, Mestre \\ https://orcid.org/0000-0001-7029-6482 \\ dmitryev14@gmail.com \\ Universidade Federal do Rio Grande do Norte $\mid$ Centro de Ciências Sociais Aplicadas \\ Natal | Rio Grande do Norte | Brasil \\ Marcos Fernando Machado de Medeiros, Doutor \\ https://orcid.org/0000-0002-7410-9290 \\ mfmedeiros@gmail.com \\ Universidade Federal do Rio Grande do Norte | Centro de Ciências Sociais Aplicadas \\ Natal | Rio Grande do Norte | Brasil \\ Manoel Veras de Sousa Neto, Doutor \\ https://orcid.org/0000-0002-9836-6873 \\ manoel.veras@uol.com.br \\ Universidade Federal do Rio Grande do Norte | Centro de Ciências Sociais Aplicadas \\ Natal | Rio Grande do Norte | Brasil
}

Recebido em 15/janeiro/2019

Aprovado em 26/fevereiro/2020

Publicado em 01/maio/2020

Sistema de Avaliação: Double Blind Review

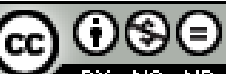

Esta obra está sob uma Licença Creative Commons Atribuição-Uso. 


\title{
RESUMO
}

Este estudo teve como objetivo verificar a aplicabilidade do modelo Life Cycle Canvas ${ }^{\circledR}$ (LCC) de gestão de projetos no contexto da aprendizagem baseada em projetos. Para tanto, fez-se uso de um estudo de caso de natureza qualitativa, exploratório e descritivo, em uma disciplina em nível de pós-graduação de um programa de mestrado de uma instituição pública de ensino superior. Do ponto de vista teórico, o alicerce para o estudo são as metodologias ativas, a aprendizagem baseada em projetos, sala de aula invertida e o modelo de gestão visual de projetos LCC. Os principais resultados foram: aumento da disponibilidade de tempo para a realização das atividades em grupo mediadas pelo docente, uso simultâneo de diversos recursos digitais, estreitamento da relação professor-aluno e aluno-aluno e flexibilização do ritmo de aprendizagem. Estes aspectos corroboram com a teoria apresentada. Por fim, o LCC atuou como um facilitador no processo de aprendizagem, dando foco à $\mathrm{ABPj}$, melhorando o desempenho tanto do docente quanto do discente.

Palavras-chave: Gestão de Projetos. Aprendizagem baseada em projetos. Sala de aula invertida. Life Cycle Canvas.

\begin{abstract}
This study aimed to verify the usability of the Life Cycle Canvas ${ }^{\circledR}$ (LCC) model of project management in the context of project-based learning. For that, a case study of qualitative, exploratory and descriptive nature was used. From the theoretical point of view, the foundation for the study are the active methodologies, the project-based learning, the flipped classroom and the methodology for the management projects Life Cycle Canvas. The main results were: increased availability of time for the group-mediated activities of the teacher, simultaneous use of several digital resources, closer teacher-student and student-student relationship and flexibility of learning pace. These aspects corroborate with the theory presented. Finally, the LCC acts as a facilitator in the learning process, focusing on projectbased learning, improving the performance of both the teacher and the student.
\end{abstract}

Keywords: Project management. Project based learning. Flipped classroom. Life Cycle Canvas. 


\section{INTRODUÇÃO}

O desenvolvimento de modelos de ensino que sejam eficientes, proporcionando um bom desempenho e alto engajamento do alunado, e ainda sejam compatíveis com o orçamento das instituições de ensino é um problema enfrentado no mundo todo. Estudiosos da educação vêm recomendando a Aprendizagem Baseada em Projetos (ABPj), como abordagem de ensino para a superação desse desafio (GHOSH, 2000; BARELL, 2006; DAVID, 2008; LABOY-RUSH, 2011; BENDER, 2015).

A ABPj é um modelo de ensino-aprendizagem que auxilia o desenvolvimento de competências e habilidades necessárias para o século 21. O aluno apreende conceitos e conteúdos ao passo que desenvolve habilidades específicas ao confrontar problemas, considerados relevantes e significativos no mundo real. Os alunos são impulsionados por questões motivadoras oriundas de situações verídicas e, mediante um trabalho colaborativo, buscam conhecimentos para elencar possíveis resoluções ao dilema proposto. Projetos concretos são realizados ao longo desse processo, desenvolvendo a criatividade, respeitando o estilo de aprendizado dos alunos e atendendo a uma prerrogativa básica da $\mathrm{ABPj}$, que é a elaboração de um produto no final. (BENDER, 2015).

O desafio fundamental dessa nova criação pedagógica é confrontar o papel do tutor, a fim de saber como supervisionar os aprendizes e também como envolvê-los na vida profissional para atingir os objetivos gerais de aprendizagem. Cargnin-Stieler et al. (2014) e Bender (2015), reconhecem a dificuldade que os alunos e professores têm em planejar e executar os projetos de ABPj. Bender (2015) evidencia ainda que os controles próprios a um projeto precisam ser feitos por meio de anotações dos alunos e não pormenoriza a maneira que esse controle se dá. Nesse contexto, identifica-se que um modelo de gestão pode auxiliar o aluno a planejar e visualizar melhor o andamento do seu projeto, o que potencialmente seria um benefício para a aprendizagem, tendo em vista que o controle dos processos que envolvem o projeto se torna um problema a menos na construção do conhecimento do aluno.

Para que seja viabilizado esse auxílio, ferramentas visuais servem de apoio ao planejamento e para simplificação do gerenciamento de projetos proporcionando uma melhor integração do gerenciamento do negócio ao gerenciamento dos projetos. Essas ferramentas facilitam a comunicação e o entendimento das partes envolvidas na gestão de projetos, além de uma interação mais dinâmica e participativa entre a equipe, quando das reuniões de acompanhamento de projeto, por exemplo (VERAS, 2016). 
Tendo em vista a necessidade de gerenciar o projeto de maneira sistematizada, de modo a não abrir mão da simplicidade dos modelos visuais de gestão de projetos, que foi criado o Life Cycle Canvas (LCC) (VERAS, 2016), permitindo o gerenciamento do ciclo de vida completo do projeto (iniciação, planejamento, monitoramento \& controle, encerramento).

Diante do exposto, a utilização de modelos de gestão visual de projetos pode permitir a gerência de maneira simplificada do ciclo de vida completo do projeto, que nesse contexto é apresentado como parte primordial da adoção da metodologia ABPj. Entretanto, o uso de modelos de gestão visual de projetos no desenvolvimento de disciplinas que utilizam o método $\mathrm{ABPj}$ não foi constatado, o que evidencia essa lacuna na literatura. Esta feita, a pesquisa se propôs a verificar a aplicabilidade do LCC no gerenciamento de projetos em sala de aula associado à sala de aula invertida.

\section{REFERENCIAL TEÓRICO}

\subsection{LIFE CYCLE CANVAS}

A despeito do aumento da complexidade, houve o crescimento de um modo de pensar que busca "descomplicar" a gestão. Nesse cenário, houve um crescimento da utilização de ferramentas e modelos visuais. Com início na abordagem Lean e baseados no uso de ferramentas e técnicas, permitem uma melhor e mais fácil visualização das informações. Por conseguinte, confere uma visão integrada por meio de quadros ou telas, podendo ser utilizado para ter uma noção holística da organização. Essa tendência pode ser notada não só na gestão como um todo, mas principalmente na Gestão de Projetos (MEDEIROS et al., 2017; VERAS, 2018).

Além de estimular a inovação, o uso de ferramentas ou modelos que têm uma característica mais visual auxilia pessoas que habitualmente não lidam com a gestão. $\mathrm{O}$ primeiro contato com algo que seja visualmente agradável é positivo, sendo evidenciado em estudos que apontam para o caráter mais amigável e livre que essas ferramentas podem trazer (ARANTES; NORMANHA FILHO, 2015).

A lógica dos modelos visuais de gestão de projetos atende ao modelo japonês de $5 \mathrm{~W} 2 \mathrm{H}$, o qual utiliza perguntas básicas para direcionar o pensamento do gestor a particularidades que são aparentemente simples, mas que podem, em alguns projetos, exigir muito mais esforço e atenção. O uso extensivo e variado desses modelos visuais já vem sendo visualizado em outras áreas como em mapeamento de processos, acompanhamento dos fluxos 
de produção, e avaliação de desempenho para gerenciar recursos (STEENKAMP, HAGEDORN-HANSEN; OOSTHUIZEN, 2017; VERAS, 2018).

Medeiros e Silva (2017) indicam que, dentre os modelos visuais analisados, o LCC foi o mais alinhado às técnicas e métodos que adotam um modelo de gerenciamento de projetos sugeridos pelos guias e modelos de boas práticas mais tradicionais. Entretanto, em uma única tela o LCC promove uma visão integrada de processos, seguindo um fluxo de atividades que contempla o ciclo de vida do projeto por completo.

O LCC se propõe a facilitar o controle das atividades inerentes ao gerenciamento de um projeto no seu ciclo de vida. Isso confere à equipe do projeto a atenção em grupos de atividades que são cruciais, como tempo, risco e custo, sem perderem recursos em detalhes supérfluos (VERAS, 2018). Essa é a ideia central do uso do modelo LCC na metodologia da ABPj.

O modelo LCC prevê o percurso completo pelas fases do projeto, ou seja, iniciação, planejamento, execução, monitoramento \& controle e encerramento (VERAS, 2018). Dessa forma ele se alinha ao padrão utilizado internacionalmente, o guia PMBOK, assim agregando rigidez metodológica à construção do projeto. Ademais, desconcentra a informação e entrega à equipe do projeto o domínio da sua gestão, trazendo, por conseguinte um maior engajamento dos envolvidos (MEDEIROS; VERAS, 2016; MEDEIROS et al., 2017; VERAS, 2018).

O LCC se diferencia dos demais modelos visuais pelo gerenciamento ao longo do ciclo de vida do projeto, que se divide em cinco grupos pelo PMI. A primeira fase, a de iniciação (Figura 1), integra o primeiro conjunto de processos de gerenciamento do PMBOK, que começa pela estruturação de uma determinada necessidade identificada, e, por conseguinte, a definição de um novo projeto ou uma nova fase de um projeto existente (PMI, 2017; MEDEIROS; ARAÚJO; OLIVEIRA, 2018).

Os processos próprios de um plano de gerenciamento e os documentos que devem ser usados para executá-lo fazem parte do Planejamento (Figura 2), pormenorizando o que deve ser realizado (PMI, 2017; MEDEIROS et al., 2017).

A fase de execução (Figura 3) traz à prática o que foi planejado de forma a alocar os dados para que sejam controlados. Essa etapa é importante para integrar pessoas e outros recursos necessários para executar o plano de gerenciamento de projetos (MEDEIROS; ARAÚJO; OLIVEIRA, 2018; PMI, 2017; VERAS, 2018). 
Figura 1 Iniciação com LCC

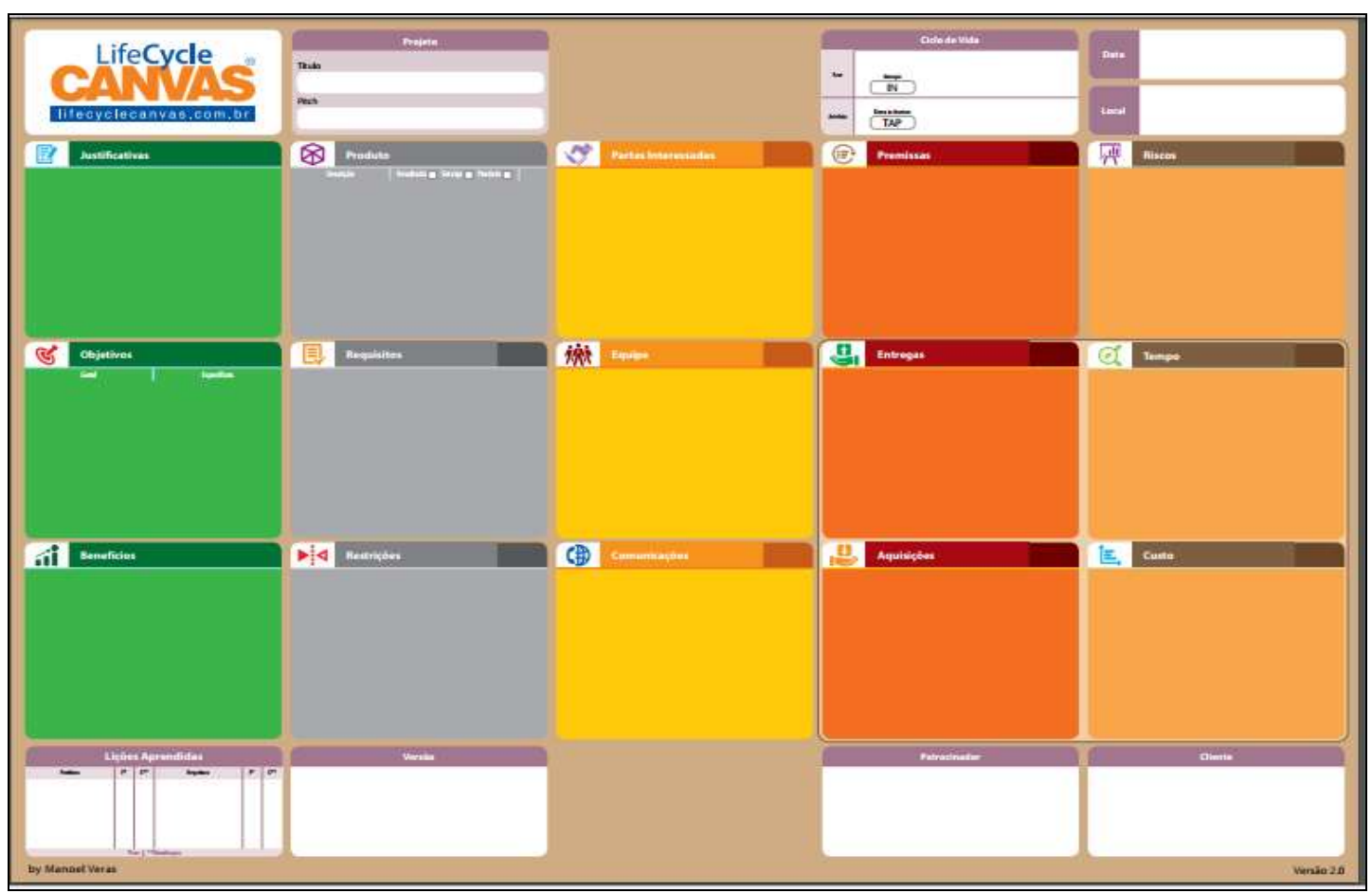

Fonte: Veras (2018).

Figura 2 Planejamento com LCC

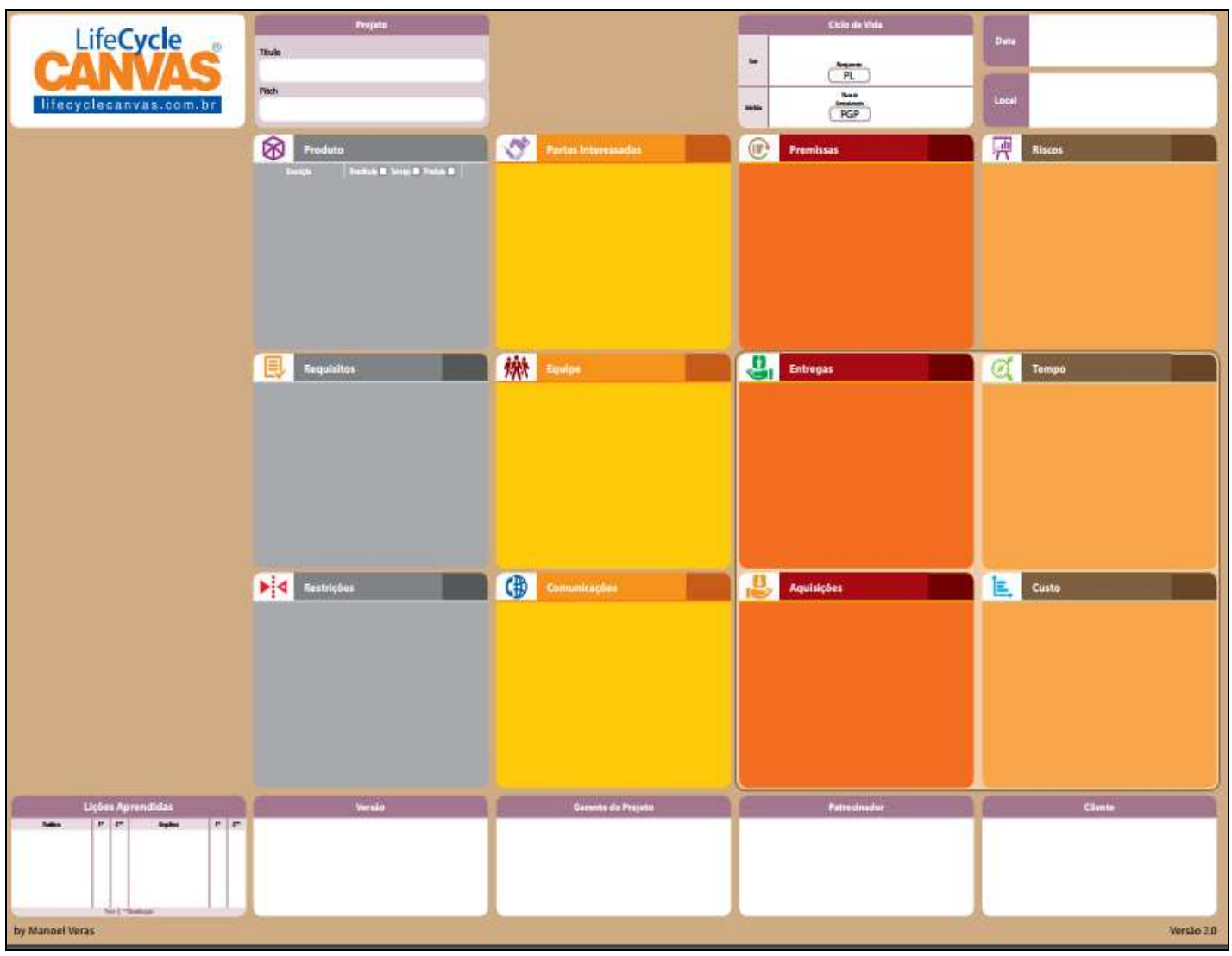

Fonte: Veras (2018). 
Figura 3 Fases de Execução e Monitoramento e Controle com LCC

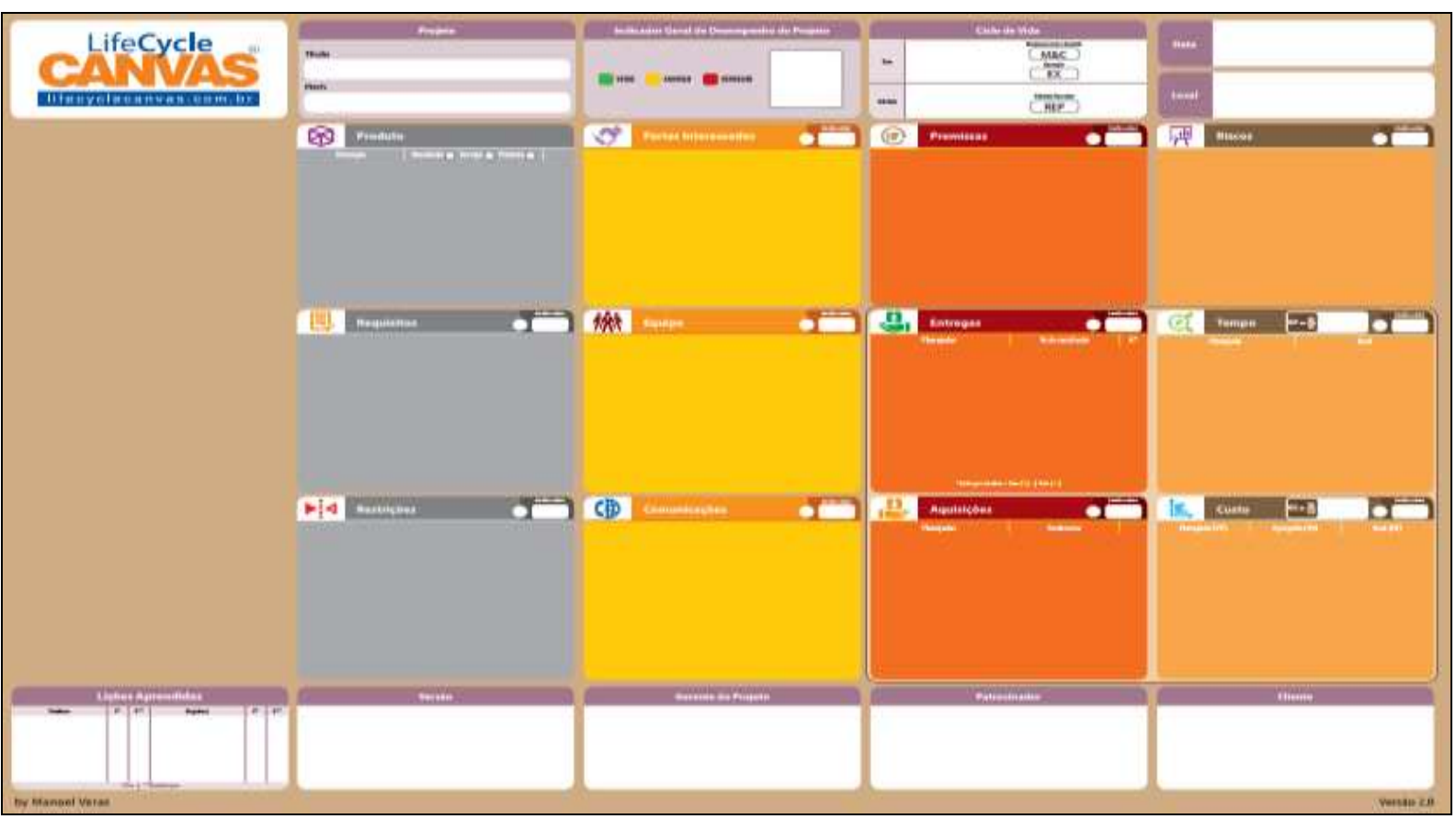

Fonte: Veras (2018).

O Encerramento (Figura 4), último grupo de processos de gerenciamento, formaliza a aceitação do produto ou serviço final do projeto ou de uma fase do projeto (VERAS, 2018).

Figura 4 Encerramento com LCC

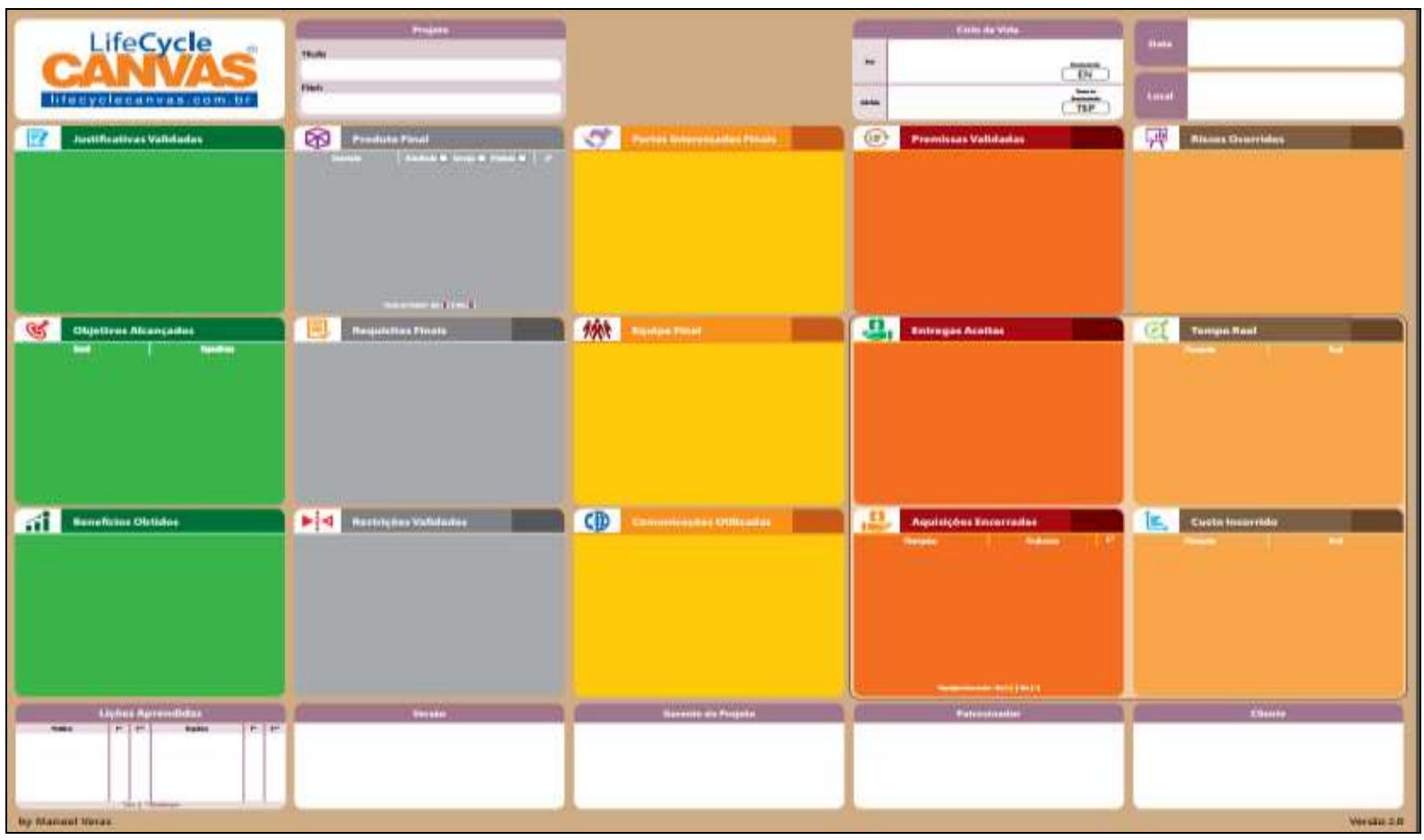

Fonte: Veras (2018). 
Em suma, o modelo LCC contempla as questões inerentes às boas práticas recomendadas pelo PMBOK, e, ao mesmo tempo, confere dinâmica e simplicidade aos processos de gerenciamento de projetos, fazendo com que a tela de projetos seja modificada ao longo do ciclo de vida.

Diante do exposto, o modelo LCC é um modelo inovador, robusto e flexível, caracterizando-se por sua aplicabilidade a projetos de qualquer tipo, qualquer abrangência ou atividade. Nesse contexto, o modelo LCC pode ser usado como facilitador do gerenciamento de atividades a serem desenvolvidas no processo de ensino-aprendizagem por meio de metodologias ativas de aprendizagem, como Aprendizagem Baseada em Problemas (ABP) ou Problem Based Learning (PBL); Aprendizagem por pares (Peer Instruction); Sala de Aula Invertida (SAI) ou Flipped Classroom (FCR); e, naturalmente, a Aprendizagem Baseada em Projetos (ABPj) ou Project Based Learning (PjBL). A seguir apresentam-se as metodologias de ensino utilizadas no presente estudo, a ABPj e SAI.

\subsection{APRENDIZAGEM BASEADA EM PROJETOS}

As metodologias ativas de aprendizagem proporcionam ao aluno vivenciar de forma antecipada problemas e situações reais da vida profissional. No método ativo, o conhecimento é construído de forma colaborativa e os alunos ocupam o centro das ações educativas, tendo como ponto de partida para construção do conhecimento seus saberes, experiências e opiniões (DIESEL; BALDEZ; MARTINS, 2017).

O uso de metodologias ativas é sugerido como um bom recurso para reduzir o presenteísmo de alunos universitários (caracterizado pela presença física e ausência de participação nas atividades acadêmicas), especialmente em conjunto ao uso de ferramentas tecnológicas (PAULO; COSTA; ANDRADE, 2018). A implantação de metodologias ativas também está associada a retenção (permanência) de aluno no ensino superior, sendo mais intensa quando há uma percepção do alunado quanto à qualidade deste novo método pedagógico. As universidades públicas poderiam explorar mais o uso de metodologias ativas de aprendizagem ao ponto em que os alunos percebessem e valorizassem a inovação nos processos de ensino, tornando-o atrativo principal de permanência do estudante e alterando a posição da gratuidade para um plano complementar (GUIMARÃES et al., 2019).

O uso de projetos, a inversão da sala de aula e a aprendizagem por meio de problemas são exemplos de metodologias ativas de aprendizagem, pois baseiam seus processos de 
aprendizado na ação do aluno. O uso pedagógico de projetos e na construção de conhecimentos foi idealizado por John Dewey, em 1897, e desenvolvido por William Kilpatrick (BARBOSA; MOURA, 2013).

Holm (2011) define a ABPj pragmaticamente como uma formação centrada no aluno que em um período definido os alunos selecionam, planejam, investigam e produzem um produto em resposta a um problema da realidade estudada.

Diversas publicações e experiências escolares referem-se ao potencial dos projetos de aprendizagem como apoio valoroso para o processo educativo, especialmente no que diz respeito à promoção de uma aprendizagem significativa, levando em consideração a aplicação em situações reais relativas ao contexto e à vida, no sentido mais amplo, que devem estar relacionadas ao objeto central do projeto em desenvolvimento (GONZÁLEZ-CARRASCO et al; HABÓK; NAGY, 2016; BARBALHO et al.; PEREIRA, BARRETO; PAZETI, 2017).

\subsection{SALA DE AULA INVERTIDA}

A sala de aula invertida (SAI) ou flipped classroom method (FCM) conecta vários conceitos educacionais, incluindo a aprendizagem ativa, significativa e colaborativa. Referese basicamente a transferir o que tradicionalmente é feito em sala de aula para casa e viceversa. Geralmente se converte a leitura ou instruções em um vídeo ou em outro formato digital, em sintonia com os atuais estudantes altamente conectados com novas tecnologias e recursos digitais. Os alunos apreendem as informações básicas em casa, registram anotações e dúvidas; e, posteriormente, são auxiliados em sala de aula a compreender os conceitos que sentem bloqueados e a aplicar o conhecimento adquirido. O tempo de sala de aula é totalmente reestruturado, sendo utilizado para a realização de atividades, atribuições práticas e ajuda direcionada, as quais devem ser obrigatórias e envolver aprendizado interativo, exceto palestras. O ganho significativo no tempo destinado à prática orientada e independente ou a atividade de laboratório com o uso de sala de aula invertida, ultrapassa 100\% de aumento, quando comparado o tempo de práticas do modelo tradicional de ensino (BERGMANN; SAMS, 2017; BISHOP; VERLEGER, 2013; ZUBER, 2015).

Outros recursos como textos impressos e áudios (podcasts) podem ser produzidos para instrumentalizar o estudante para a discussão e a solução de problemas, não existindo a necessidade de uma grande produção de material, mas sim que esse material seja elaborado com o intuito principal de engajar os estudantes, sem provocar uma sobrecarga de 
informações (SHARMA et al, 2015). A integração com os alunos por meio de um ambiente virtual, como Moodle ou o EdModo, ou o uso de blogs para fornecer material aos alunos, associados a um espaço em que os estudantes possam colocar suas anotações também são opções de interação com os alunos (BOLLELA; 2017).

A aplicação do método é alicerçada em seus quatro pilares básicos: 1-Flexible (F), o processo deve ser flexível e favorecer o uso de diferentes modos de ensino-aprendizagem; 2Learning (L), o aprendizado deve ser centrado no estudante e na sua relação com os pares; 3Intentional teaching actions (I), o professor deve ter intencionalidade nas atividades realizadas e propostas para os alunos; 4-Professional Educator $(\mathbf{P})$, o professor deve avaliar e dar feedbacks aos alunos, bem como refletir e avaliar sua própria prática. (FLN, 2014).

A inversão da sala de aula apresenta diversas vantagens. Permite explorar os recursos digitais; aproximar a linguagem dos estudantes da atualidade, os quais podem estar conectados a diferentes dispositivos digitais e realizando multitarefas simultaneamente; flexibilizar o ritmo de aprendizagem de acordo com a necessidade de cada estudante; auxiliar estudantes que enfrentam dificuldades; ajudar alunos com diferentes habilidades a se superarem, considerando necessidades especiais; intensificar a interação aluno-professor e aluno-aluno; personalização da aprendizagem; facilita o gerenciamento da sala de aula, pois os alunos estão envolvidos com as atividades e ficam menos propensos a se dispersar. Adicionalmente, torna a aula mais transparente, pois o material está disponível para todos os interessados na internet (BERGMANN; SAMS, 2017).

A literatura aponta melhoria na aprendizagem dos alunos de ensino superior em distintas áreas, tanto em nível de graduação como em pós-graduação. Pavanelo e Lima (2017), ao utilizar o método para o desenvolvimento de uma disciplina de cálculo, dos cursos de Engenharia do Instituto Tecnológico de Aeronáutica, identificou como pontos positivos o uso do tempo de sala de aula para as atividades interativas em grupo e o melhor desempenho dos alunos nas avaliações, corroborando com evidências identificadas por Bishop e Verleger (2013). Forte evidência atual, dada por estudo utilizando meta análise acerca da educação das profissões de saúde, sugere que a abordagem de SAI rende melhoria significativa na aprendizagem dos alunos em comparação com os métodos tradicionais de ensino (HEW; LO, 2018).

Associando o LCC à ABP e a SAI, o ponto de partida é o preenchimento da tela Educação (Figura 5) que contempla, entre outras informações, a âncora e a questão motriz. 
Figura 5 Utilização de SAI com LCC

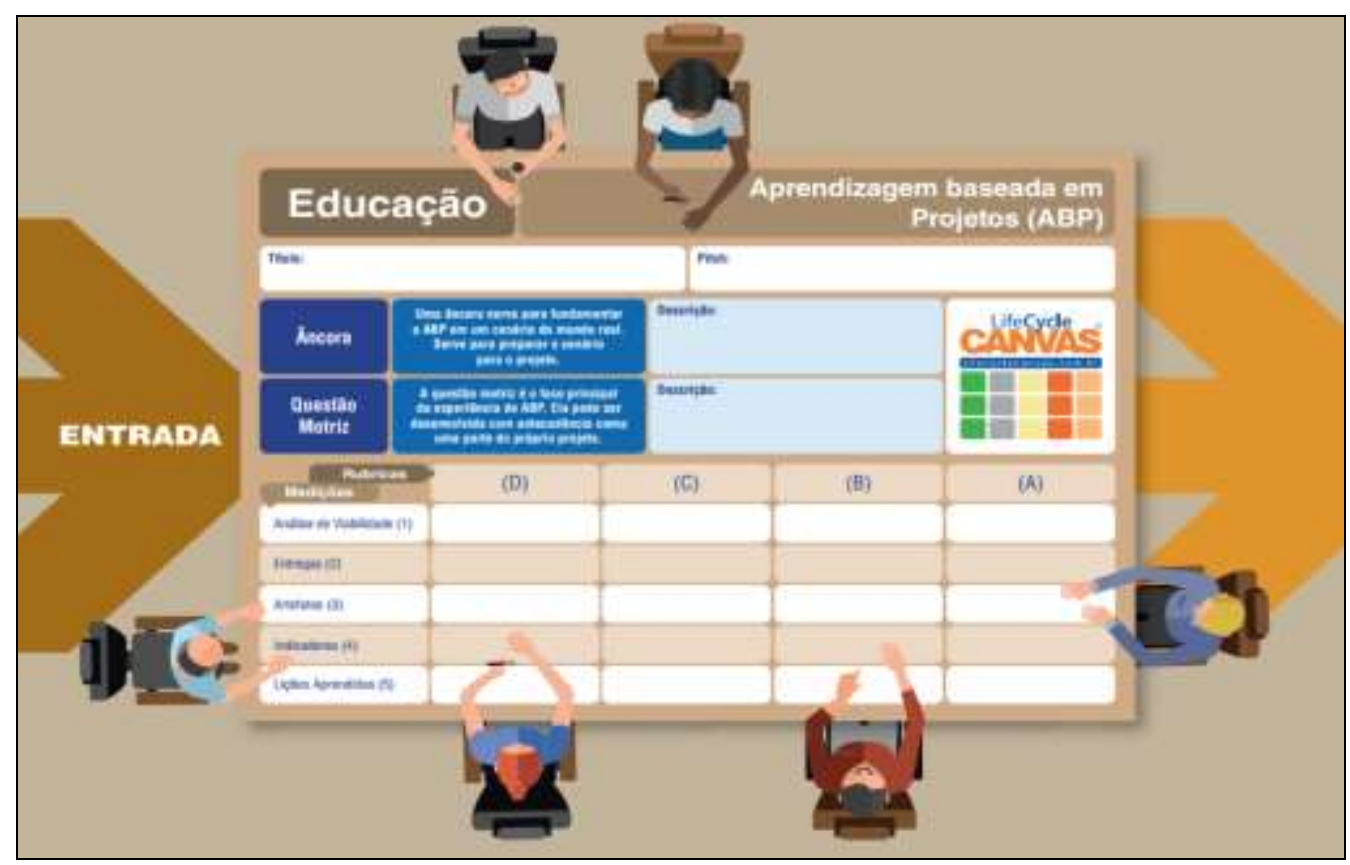

Fonte: Elaborado pelos autores, 2018.

A partir daí o ciclo da gestão de projetos é realizado (Figura 6), passando pelas etapas de iniciação, planejamento, monitoramento, controle e execução, encerramento.

Figura 6 Utilização de SAI no ciclo de vida dos projetos

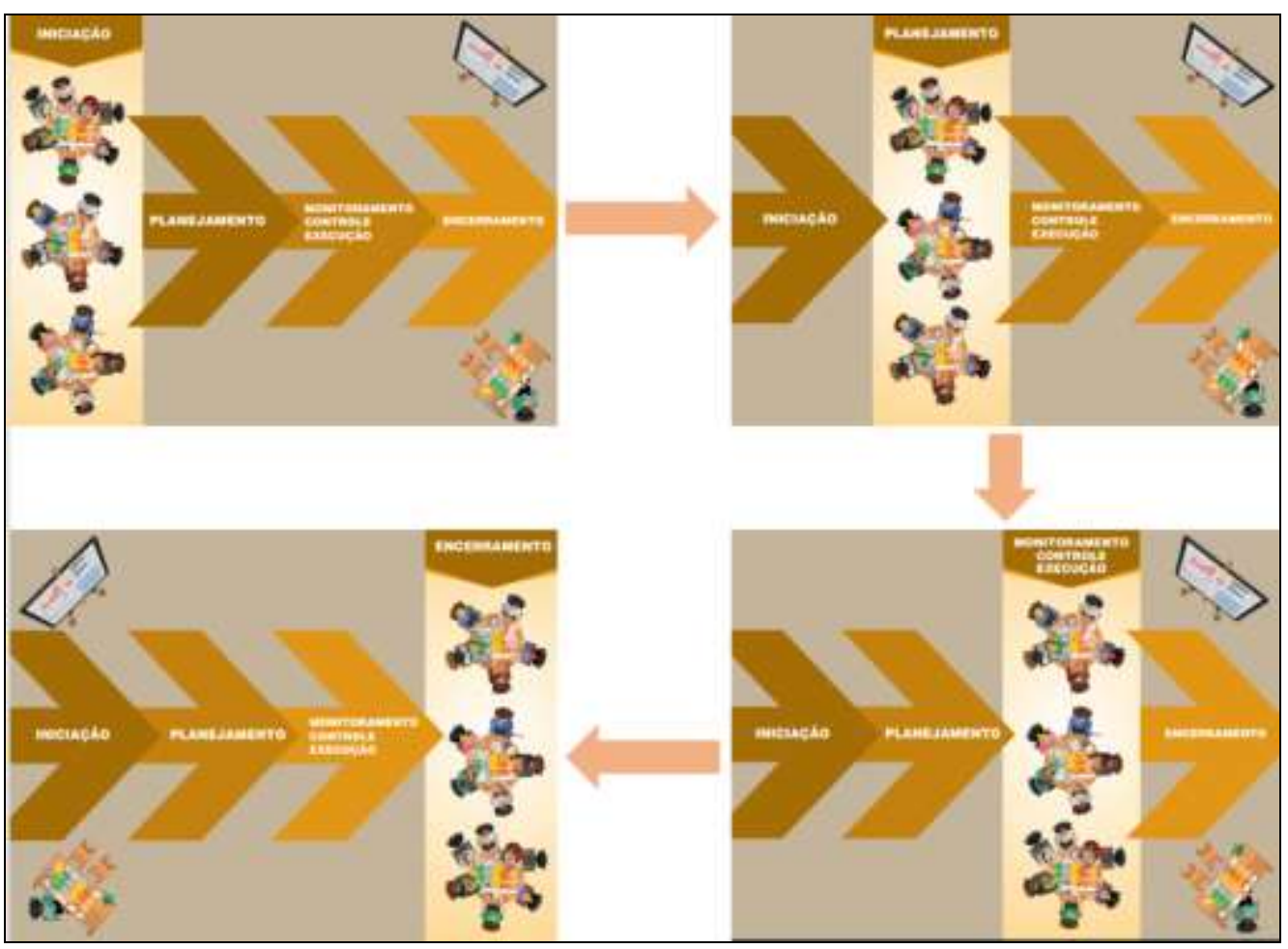

Fonte: Elaborado pelos autores, 2018. 
Finaliza-se o processo avaliando as etapas de realização do projeto e preenchendo a tela de lições aprendidas (Figura 7) para cada projeto, onde os resultados dos projetos são avaliados.

Figura 7 Utilização de SAI com LCC

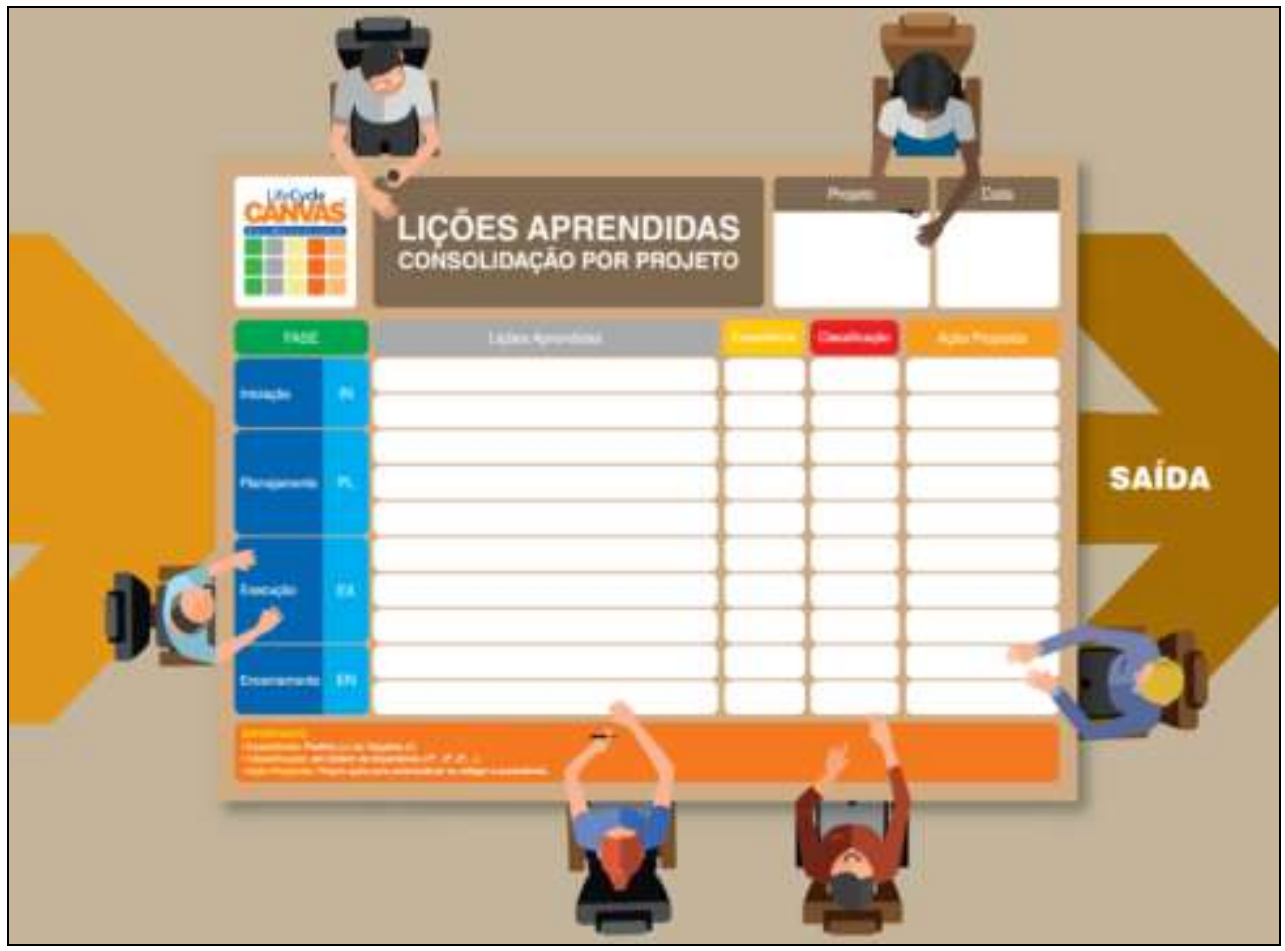

Fonte: Elaborado pelos autores, 2018.

Esse desenho didático-metodológico ainda que aplicado como modelo híbrido (associando o ensino tradicional e sala de aula invertida) pode proporcionar uma adequada interação entre professores e alunos, assim como entre os próprios alunos, proporcionando uma aprendizagem significativa utilizando os recursos tecnológicos da internet (FRANCISCO; OLIVEIRA, 2016).

Posto que, os conceitos aos quais o estudo perpassa foram firmados, e na perspectiva de compreender melhor os processos do trabalho proposto seguimos à próxima seção que trata dos procedimentos e caracterizações metodológicas dessa pesquisa que associa o uso do modelo LCC a práticas ativas de ensino-aprendizagem.

\section{PROCEDIMENTOS METODOLÓGICOS}

Visando alcançar o objetivo proposto pelo estudo - verificar a aplicabilidade de um modelo de gestão visual do ciclo vida de projetos, o modelo Life Cycle Canvas ${ }^{\circledR}$, no contexto 
do gerenciamento das atividades de aprendizagem baseada em metodologias ativas de aprendizagem - optou-se por fazer pesquisa-ação, de natureza qualitativa, com caráter exploratório e descritivo, por meio de estudo de caso.

O estudo foi realizado em uma disciplina em nível de pós-graduação de um programa de mestrado de uma instituição pública de ensino superior. A escolha do caso foi por conveniência, em decorrência da parceria estabelecida entre docentes dos cursos de Administração e Educação em prol da aplicação do modelo LCC na disciplina “Aprendizagem Baseada em Projetos". No entanto, a relevância é significativa em virtude do caráter inovador dessa experiência, que buscou apresentar uma solução prática para que os próprios alunos possam assumir o papel ativo no gerenciamento de seus projetos no meio acadêmico. Além disso, a pesquisa representa o estudo piloto para uma dissertação de mestrado e uma tese de doutorado, ambas do programa de Pós-graduação em Administração da mesma instituição.

A pesquisa foi desenvolvida de abril a julho de 2017. Os pesquisadores participaram desde o planejamento da disciplina, acompanharam a realização das atividades práticas em sala de aula e atuaram como facilitadores no processo de preenchimento da tela de apoio do LCC ${ }^{2}$, assistiram à apresentação final dos grupos de alunos e acompanharam a avaliação realizadas pelos professores quanto ao desenvolvimento dos alunos e a adequação do modelo à disciplina.

O processo de coleta de dados foi realizado por meio de observação participante, análise documental e aplicação de questionário. Inicialmente os investigadores coletaram dados através da observação e da interação com o objeto de estudo. Durante esta etapa, os pesquisadores se concentraram em identificar como os quatro grupos conduziram o preenchimento da tela do LCC, visando entender o comportamento dos alunos no processo de gerenciamento de projetos na área de educação, bem como registrar as facilidades e dificuldades enfrentadas.

Além disso, a triangulação, apontada por Flick (2009) como fator de qualidade na pesquisa qualitativa, também foi observada no estudo. Foram realizadas as triangulações de método, triangulação de investigadores e dados. Foram utilizados dois métodos qualitativos e um quantitativo que busca a circularidade e validação, além de gerar diferentes fontes de dados, ademais dois dos investigadores estavam envolvidos na observação participante o que dá diferentes perspectivas do ponto de vista analítico. 
A análise documental utilizou os artefatos produzidos pelos alunos (telas principais do LCC preenchidas) e pelos professores (tela de rubrica de avaliação). De forma a cumprir com o objetivo proposto no estudo, a análise documental também examinou como os grupos assimilaram a ideia proposta de estabelecer um processo gerenciamento de projetos de educação e como o uso do modelo LCC® contribuiu para tornar o processo de inovação uma ação efetiva. Essa etapa verificou basicamente a consistência e a coesão a partir de uma análise mais detalhada dos documentos preenchidos por cada um dos grupos relativos aos produtos de inovação propostos.

O questionário aplicado com os professores foi composto por 8 questões, 2 fechadas (Escala Likert com 5 pontos) e 6 abertas, subdivididas em quatro seções que buscaram contemplar a percepção dos professores quanto a: 1-O LCC propriamente dito, 2-A associação da gestão de projetos às características $\mathrm{ABPj}, 3-\mathrm{A}$ associação da gestão de projetos e do LCC à metodologia $\mathrm{ABPj}$ e; por fim, o item mais importante 4- A associação do LCC ao gerenciamento de projetos em $\mathrm{ABPj}$. $\mathrm{O}$ questionário foi enviado por correio eletrônico e o envio foi comunicado aos respondentes por aplicativo de mensagens de texto.

Quanto aos resultados, os dados provenientes das questões fechadas do questionário foram descritos textualmente e as respostas dadas às questões abertas foram parcialmente transcritas. Visando não identificar as respostas individuais dos entrevistados, os mesmos foram codificados aleatoriamente como Professor 1 (P1) e Professor 2 (P2).

Por se tratar de uma pesquisa-ação, a análise de dados à luz da literatura unida a análise de documentos (os artefatos gerados a partir do uso do LCC) e as observações registradas durante o processo, constituíram importantes instrumentos para obter os resultados e as conclusões desse estudo.

\section{RESULTADOS E DISCUSSÕES}

\subsection{O PROCESSO DE UTILIZAÇÃO DA SAI-ABPJ GERENCIADA PELO LCC}

A primeira etapa correspondeu ao planejamento da disciplina, que compreendeu o aprofundamento do conhecimento acerca do referencial teórico (SAI, ABPj, LCC), definição de estratégias e recursos de ensino aprendizagem, planejamento de cronograma, seleção da bibliografia básica, discussão acerca do método e rubrica de avaliação. Foi realizada em abril de 2017, tendo 2 reuniões presenciais entre os professores, com a presença dos pesquisadores. 
A segunda etapa foi a realização da disciplina, que ocorreu durante os meses de maio e junho, com carga horária total de 30 horas, tendo encontros presenciais quinzenais aos finais de semana (sexta e sábado). A metodologia usada foi a Sala de Aula Invertida-SAI (Figuras 5, 6 e 7), associada a práticas de ABPj gerenciadas pelo LCC. No primeiro dia de aula, a metodologia de ensino, as estratégias, o cronograma e a bibliografia básica para estudo foram apresentados para os alunos. No desenvolvimento da SAI, a atividade de casa correspondeu a leitura de material didático acerca da $\mathrm{ABPj}$ e do $\mathrm{LCC}$, disponibilizado por meio do ambiente virtual habitualmente utilizado na instituição de ensino.

A prática em sala utilizou como estratégia a $\mathrm{ABPj}$, através da qual cada grupo de alunos desenvolveu um projeto na área de educação e entregou um produto. Como parte da ABPj, utilizou-se um vídeo "âncora" para fundamentar um cenário do mundo real, dando ênfase a importância do assunto geral proposto à turma (Educação na atualidade). Em seguida, os grupos de alunos se reuniram e definiram a "questão motriz", ou seja, o tema específico que seria o foco principal da experiência de ABPj de cada grupo, a saber: 1- Como inserir jogos digitais no ensino da Administração de Recursos Financeiros?; 2Aperfeiçoamento do conhecimento de Tecnologias de Informação e Comunicação (TIC) para um grupo de terceira idade usando como foco motivacional as experiências de vida dos próprios alunos; 3- Sala de Aula Invertida: como fazer?; 4-Escola e Comunidade, construindo uma cultura de paz. Também foram realizadas miniaulas (com duração de 15 a 20 minutos) sobre o gerenciamento de projetos com uso do LCC, acompanhando a realização do ciclo de vida (Iniciação, Planejamento, Execução, Monitoramento \& Controle, Encerramento) dos projetos que foram desenvolvidos pelos alunos.

O desenvolvimento das atividades práticas em sala de aula foi acompanhado pelos professores e pesquisadores, sendo realizados feedbacks para os alunos. As dúvidas foram esclarecidas presencialmente e, quando fora do horário de aula, por aplicativo de mensagens de texto. O ritmo de aprendizagem de cada grupo foi respeitado, tendo-se maior atenção e acompanhamento para um grupo específico que demonstrou tal necessidade, seguindo preconização da $\mathrm{ABPj}$. Conforme previsto no cronograma, todos os grupos realizaram seus projetos e, ao final da disciplina, apresentaram os resultados da experiência e entregaram os produtos elaborados: livro, e-book, jogo digital com guia de uso, e material publicitário para campanha educativa.

Identificou-se que os 4 pilares básicos da metodologia sala de aula invertida, 
referenciados por FLN (2014) e Wang (2017), foram respeitados no presente estudo: ambiente flexível, cultura de aprendizagem centrada no estudante, conteúdo intencional, e educadores preparados. A principal vantagem da sala de aula invertida observada nessa experiência foi o aumento da disponibilidade de tempo para realização de atividades interativas em grupo com a mediação do professor, o que permitiu outras vantagens como o uso simultâneo de diversos recursos digitais; a aproximação com os alunos e o estreitamento da relação professor-aluno e aluno-aluno; a maior disponibilidade de tempo para os alunos que enfrentaram dificuldades pessoais ou de aprendizagem; e a flexibilização do ritmo de aprendizagem, respeitando o prazo final para a conclusão do projeto; benefícios esses que corroboram com os apontamentos da literatura atual (BERGMANN; SAMS, 2017; PAVANELO; LIMA, 2017).

Os alunos passaram por todo o ciclo de um projeto: iniciação, planejamento, execução, monitoramento e controle, e, por fim o encerramento. Para tanto os alunos preencheram as 4 telas principais do LCC, a tela de lições aprendidas e a tela do LLC-Educação (Figuras 1, 2, 3, 4, 5 e 7). Foi observado que houve um forte envolvimento da turma com o conteúdo, contribuindo para a aprendizagem significativa dos alunos e um bom desempenho nas avaliações realizadas, sendo estes itens verbalizados pelos próprios alunos ao final da disciplina. Tais resultados ratificam os achados de Moran e Milsom (2015), Foster e Stagl (2018), Bishop e Verleger (2013) e Hew e Lo (2018).

As características básicas da ABPj, elencadas por Bender (2015), foram evidenciadas pelos pesquisadores no presente estudo: seleção de uma âncora; definição de questão motriz pelos alunos; realização de trabalho cooperativo em equipe; investigação e inovação nas tarefas do projeto; reflexão; valorização da voz e escolha do aluno ao longo do projeto; feedback e revisão dos professores e colegas; e apresentação pública dos resultados. O relato dos alunos apontou o uso de metodologia ativa como fator primordial da aprendizagem e do desempenho na disciplina. Percepção similar foi apontada por Escrivão Filho e Ribeiro (2008) quando utilizaram um outro tipo de metodologia ativa, a Aprendizagem Baseada em Problemas (ABP ou PBL), no ensino da administração. Os autores identificaram que comparado às abordagens instrucionais tradicionais, o uso da metodologia ativa apresentou-se vantajoso mesmo quando adotado parcialmente. Além disso, ressaltaram o estreitamento da relação aluno-aluno e professor-aluno como um fator afetivo importante.

Por meio do acompanhamento do gerenciamento dos projetos e do preenchimento dos artefatos do LCC, os pesquisadores elencaram os conceitos de gestão de projetos que geraram 
mais solicitação de esclarecimentos: requisitos, restrições, premissas, riscos, produto e entregas. No entanto, pôde-se identificar que os conceitos foram sendo apreendidos e utilizados pelos grupos durante o preenchimento de telas e a realização da gestão de seus respectivos projetos aplicados a situações reais de suas práticas profissionais. Esse achado alinha-se com a evidencia científica acerca da necessidade de associar os conteúdos/atividades de sala de aula à experiência profissional dos estudantes, visando a mobilização e o desenvolvimento sistemático de competências, a qual foi apontada como uma das considerações mais frequentes num estudo sobre desenvolvimento de competências, no contexto de programas de pós-graduação em formação gerencial (RUAS; COMINI, 2007).

Adicionalmente, os alunos identificaram o LCC como facilitador do gerenciamento dos projetos desenvolvidos. As observações dos pesquisadores endossam que o LCC se apresentou como um instrumento visual de simples aplicação devido ao uso de telas e, ao mesmo tempo, muito robusto por se apoiar metodologicamente em guias da área de gestão de projetos reconhecidos internacionalmente. Facilitou a relação das partes interessadas, dando uma visão integrada, clara e global do projeto. Demonstrou-se escalável, servindo para os distintos tamanhos de projetos desenvolvidos em sala de aula, e flexível, sendo usado por pessoas com diferentes níveis de conhecimento prévio acerca da área gestão de projetos. Seu grande diferencial, quando comparado a outras ferramentas de gestão, foi o seu exclusivo estilo dinâmico, que permite gerenciar o ciclo de vida inteiro dos projetos, conforme proposto por Veras (2016) e Medeiros e Veras (2016).

A terceira etapa foi a avaliação dos alunos, realizada utilizando uma tela específica contendo rubricas, desenvolvida especificamente para contemplar a aplicação do LCC à ABPj. Foram avaliados os seguintes quesitos: análise da viabilidade e desenvolvimento do projeto, qualidade e prazos das entregas/produto final, qualidade e prazos de conclusão do preenchimento dos artefatos/telas LCC, registro das lições aprendidas; além da avaliação do envolvimento dos grupos com as práticas educativas aplicadas (SAI e ABPj), o desenvolvimento do projeto em consonância com o assunto proposto e a apresentação para a turma. A avaliação ocorreu de forma contínua, tendo seu valor atribuído ao final da disciplina após a realização de uma reunião entre professores. Apenas um grupo não atingiu a pontuação mínima necessária para aprovação no quesito preenchimento de artefatos (telas LCC). Considerando a necessidade de acompanhamento mais específico para esse grupo, conforme preconiza a metodologia ABPj (BENDER, 2015), os alunos tiveram suas dúvidas esclarecidas 
e foram orientados a preencher novamente as telas e entregar para uma nova avaliação. Ao final, todos os alunos foram aprovados.

A quarta e última etapa, ocorrida após a conclusão das atividades com os alunos, em julho de 2017, foi a avaliação da disciplina, realizada pelos professores. Os mesmos reconheceram a contribuição do LCC para o gerenciamento de projetos na área de educação, identificando-o como um modelo de gestão visual simples, robusto, flexível, escalável e dinâmico, conforme proposto pela literatura (VERAS, 2016). A metodologia de Sala de Aula Invertida, com uso de Aprendizagem Baseada em Projetos gerenciada pelo Life Cycle Canvas ${ }^{\circledR}$ foi avaliada como uma experiência positiva e, por isso, foi incorporada à disciplina e será replicada com as próximas turmas, demonstrando a adesão dos professores. Resultado positivo com continuidade da prática pedagógica também foi relatado por Escrivão Filho e Ribeiro (2008) acerca do uso de metodologia ativa no curso de Administração, evidenciando a satisfação docente, o estímulo ao aperfeiçoamento profissional do professor mediante os desafios intelectuais que emergiram durante o processo de ensino-aprendizagem e a implementação de um novo modo de ensinar-aprender após a realização da pesquisa.

Mediante observação verificou-se que os conceitos básicos acerca de SAI, ABPj e LCC foram apreendidos durante o processo de aprendizagem ativa, uma vez que a execução e gerenciamento das atividades interativas foram realizados com sucesso por todos os grupos da turma, tendo a entrega do produto final realizada de acordo com os requisitos, prazos e custos pré-estabelecidos pelos próprios alunos.

\subsection{PERCEPÇÃO DOS PROFESSORES ACERCA UTILIZAÇÃO DO LCC COM SAI- ABPJ}

Em um contexto geral, os dois professores respondentes do questionário identificaram que o LCC influencia positivamente as características básicas da ABPj. No entanto, um dos professores não considera que o LCC tenha tido qualquer influência sobre as definições da âncora e questão motriz, enquanto o outro professor considerou que houve alguma relevância. Essa relação acaba se invertendo quando questionados sobre a influência na voz e escolha do aluno, quando o professor que desconsiderou a influência do LCC nas partes iniciais analisou como alta a capacidade do LCC de melhorar essa característica e o outro professor alegou ter alguma capacidade.

O fato de um dos professores respondentes não ser da área de Administração, traz uma perspectiva mais acurada sobre a usabilidade do LCC em contextos em que os usuários não 
estão familiarizados. Este professor evidenciou que apesar do gerenciamento proporcionar um maior controle ao projeto, alguns conceitos inerentes à área de gestão de projetos podem ser inicialmente assimilados de maneira equivocada, sendo necessário algum tempo e a orientação do professor/facilitador para serem de fato apreendidos. Isso fica claro quando um dos professores atribui uma alta nota à influência no estabelecimento da estratégia, enquanto o outro se julga incapaz de opinar.

Os professores afirmaram ainda, por meio do questionário, que a associação da fundamentação teórica da área gestão de projetos (GP) à metodologia $\mathrm{ABPj}$ permitiu a otimização da aplicação de conhecimentos, habilidades, ferramentas e técnicas às atividades do projeto em educação a fim de atender seus requisitos. Justificaram que a associação "torna o processo simples e intuitivo" (P1) e que "a exigência de objetividade necessária ao desenvolvimento de um projeto, com previsões claras, etapas consistentes, faz com que não se perca o norte das ações necessárias ao bom desempenho do processo” (P2). Além disso, apontam que "a fundamentação fornece elementos norteadores fundamentais e visíveis a execução, o que facilita a revisão e o acompanhamento das ações, sem desvios, além de possibilitar que não se perca de vista o resultado/produto final." (P2)

O uso do modelo LCC foi considerado por ambos professores como facilitador da aplicação da metodologia Aprendizagem Baseada em Projetos, pois "simplifica, engaja, orienta" a gestão dos projetos e "de forma simples, ele [LCC] sinaliza que projetos possuem ciclos de vida" (P1). P2 descreve que as telas do LCC são "um dispositivo claro, objetivo e que fornece as informações precisas e necessárias ao acompanhamento" do projeto. Complementa descreve que "a proposta visual do LCC é extremamente útil para o acompanhamento do projeto, uma vez que possibilita visualizar de uma única vez todas as etapas do projeto (P2). Afirma ainda que o uso do LCC elucida os pontos críticos do projeto que merecem revisão, uma vez que proporciona a identificação de "coerências/incoerências, rupturas e sistematicidade das etapas".

O engajamento da equipe e o uso do LCC foram ressaltados como basilares para o sucesso dos projetos desenvolvidos. A transparência das informações foi percebida como um ponto fundamental, por permitir a compreensão detalhada do projeto como um todo; bem como, a identificação das inconsistências que poderiam ter um impacto negativo nos resultados. Nesse sentido, o professor P2 expõe "foi premente o envolvimento do grupo, a 
compreensão da relação entre as partes que compõem o projeto, sendo o ponto alto a visibilidade proporcionada pelo LCC".

Para os professores, o LCC apresentou contribuições importantes para o gerenciamento de projetos realizados pela metodologia $\mathrm{ABPj}$, sendo elas: 1-Rapidez na elaboração do projeto; 2-Percepção da intrínseca relação entre o problema, os objetivos, as entregas e o produto final; 3-Definição e distribuição de responsabilidades; 4-Simplificação da gestão de projetos; 5-Trilha e sequência para a realização de um projeto bem sucedido; 6Foco em resultados.

Como dificuldades decorrentes do uso do LCC para o suporte de gerenciamento dos projetos implementados por meio da metodologia $\mathrm{ABPj}$, um dos professores afirmou não identificar nenhuma dificuldade. O outro professor elencou dois pontos: 1-O desconhecimento dos alunos em relação a alguns termos utilizados na área de gestão de projetos (como por exemplo, entrega e requisitos); e 2-A necessidade de adaptação das telas (substituição do termo a um patrocinador).

Diante do exposto, os professores apresentaram uma percepção positiva em relação ao resultado do uso do LCC na gestão de projetos de $\mathrm{ABPj}$, destacando a necessidade do desenvolvimento do conhecimento acerca dos conceitos da área de gestão de projetos para a realização da prática.

\section{CONSIDERAÇÕES FINAIS}

Os conceitos básicos acerca de SAI, ABPj e LCC foram aplicados pelos professores durante a realização da disciplina, proporcionando a compreensão desses conceitos pelos alunos por meio de mini-aulas teóricas e atividades práticas de elaboração dos projetos que perpassaram todas as fases do o ciclo de vida do projeto.

O Life Cycle Canvas proporcionou a estrutura de gerenciamento de projetos para as práticas de ensino na perspectiva da Aprendizagem Baseada em Projetos. O LCC agiu como auxiliar nesse ambiente educacional, proporcionando aos alunos a iniciação e planejamento detalhado de um projeto; a execução de um projeto real por completo, o monitoramento mais preciso; a tomada de decisões para o controle com mais segurança, dada a clareza das informações, o engajamento da equipe e a comunicação facilitada pelo uso de telas específicas para cada fase do projeto; bem como o encerramento adequado e o registro de lições aprendidas. O LCC contribuiu para o aprendizado do aluno e o desenvolvimento de 
suas competências, tendo como foco a realização de projetos para resolução de problemas reais.

A metodologia de Sala de Aula Invertida, com uso de Aprendizagem Baseada em Projetos gerenciada pelo Life Cycle Canvas ${ }^{\circledR}$ foi avaliada como uma experiência positiva. Os professores sustentaram que o LCC influenciou positivamente as características básicas da $\mathrm{ABPj}$ e apontaram que a associação da fundamentação teórica da área gestão de projetos à metodologia ABPj permitiu a otimização da aplicação de conhecimentos, habilidades, ferramentas e técnicas às atividades do projeto em educação a fim de atender seus requisitos.

Os alunos reconheceram o LCC como facilitador do gerenciamento dos projetos desenvolvidos. O envolvimento da turma com o conteúdo contribuiu para a aprendizagem significativa dos alunos, evidenciada pelo bom desempenho nas atividades e avaliações realizadas. O LCC demonstrou contribuições importantes e de simples aplicação no gerenciamento dos projetos realizados.

Por se tratar de uma pesquisa-ação realizada com apenas uma turma de pós-graduação em uma instituição de ensino público, atribui-se essa limitação ao estudo. Nesse contexto, como forma de ratificar os resultados encontrados, reconhece-se a necessidade de replicar a pesquisa com diferentes grupos de pessoas e em diferentes ambientes de ensinoaprendizagem, o que representa as perspectivas futuras de novos estudos a serem realizados por este grupo de pesquisadores.

Diante do exposto, o auxílio que o LCC confere à gestão do projeto da $\mathrm{ABPj} \mathrm{em} \mathrm{si}$, proporciona condições para que os alunos disponham mais tempo e esforço para as atividades referentes à cognição e aprendizagem, contribuindo para a otimização do processo de aprendizado. Sendo assim, conclui-se que o LCC, subsidia a gerência de projetos em educação, de forma abrangente e sem perder a simplicidade necessária nesse contexto; sendo, portanto, aplicável no contexto da ABPj.

\section{REFERÊNCIAS}

ARANTES. S. S; NORMANHA FILHO, M. A. Metodologias ativas no ensino de administração: aplicação da aprendizagem baseada em projeto no desafio de pesquisar organizações do terceiro setor. Administração de Empresas em Revista, v. 14, n. 15, p. 219-237, 2015.

BARBALHO, S. C. M. et al. A Project Based Learning approach for Production Planning and Control: analysis of 45 projects developed by students. Production, v. 27, n. SPE, 2017. 
BARBOSA, E. F.; MOURA, D. G. Metodologias ativas de aprendizagem na educação profissional e tecnológica. Boletim Técnico do Senac, v. 39, n. 2, p. 48-67, 2013.

BARELL, J. F. Problem-based learning: An inquiry approach. Corwin Press, 2006.

BENDER, W. N. Aprendizagem baseada em projetos: educação diferenciada para o século XXI. Penso Editora, 2015.

BERGMANN, J.; SAMS, $A$. Sala de aula invertida: uma metodologia ativa de aprendizagem. Rio de Janeiro: LTC, 2017.

BISHOP, J. L; VERLEGER, M. A. The flipped classroom: A survey of the research. In: ASEE national conference proceedings, Atlanta, GA. p. 1-18, 2013.

BOLLELA, V. R. Sala de Aula Invertida na Educação para as Profissões de Saúde: Conceitos Essenciais para a Prática. Revista Eletrônica de Farmácia, v. 14, n. 1. p. 39-48, 2017.

CARGNIN-STIELER, M. et al. The importance of teamwork in the case of an interdisciplinary team of teachers from the first year of an engineering course. In:

Proceedings of the Sixth International Symposium on Project Approaches (PAEE2014). p. 67.1-67.9, 2014.

DAVID, J. L. Project-based learning. Educational Leadership, v. 65, n. 5, p. 80, 2008.

DIESEL, A.; BALDEZ, A.L.S.; MARTINS, S.N. Os princípios das metodologias ativas de ensino: uma abordagem teórica. Revista Thema. v. 14, n. 1. p.268-288, 2017

ESCRIVÃO FILHO, E.; RIBEIRO, L.R.C. Inovando no ensino de administração: uma experiência com a aprendizagem baseada em problemas (PBL). Cad.EBAPE.BR, Rio de Janeiro, v. 6, n. spe, p. 01-09, 2008.

FLICK, U. Qualidade na pesquisa qualitativa: coleção pesquisa qualitativa. Bookman, 2009.

FLN, Flipped Learning Network. The four pillars of FLIP. 2014. Disponível em: $<$ http://flippedlearning.org/wp-content/uploads/2016/07/FLIP_handout_FNL_Web.pdf $>$. Acesso em: 10 dez. 2017.

FOSTER, G.; STAGL, S. Design, implementation, and evaluation of an inverted (flipped) classroom model economics for sustainable education course. Journal of Cleaner Production, v. 183, p. 1323-1336, 2018.

FRANCISCO, C. N. P.; OLIVEIRA, R. S. Práticas pedagógicas digitais: o Facebook e a sala de aula invertida na formação dos alunos do ProfLetras. Revista Odisseia, v. 1, n. 2, p. 48-61, 2016.

GHOSH, P. Problem-based learning. Buzzle. 2000. Disponível em:

$<$ www.buzzle.com/articles/problem-based-learning.html $>$. Acesso em: 17 de junho de 2017. 
GONZÁLEZ-CARRASCO, M. et al. The development of professional competences using the interdisciplinary project approach with university students. Journal of Technology and Science Education, v. 6, n. 2, p. 121-134, 2016.

GUIMARÃES, J.C.F. et al. A influência da inovação no ensino, qualidade e comprometimento sobre a retenção de alunos no ensino superior. Revista Gestão Universitária na América Latina - GUAL, Florianópolis, p. 249-269, 2019.

HABÓK, A.; NAGY, J. In-service teachers' perceptions of project-based learning. SpringerPlus, v. 5, n.1, p.83, 2016.

HEW, K. F.; LO, C. K. Flipped classroom improves student learning in health professions education: a meta-analysis. BMC medical education, v. 18, n. 1, p. 38, 2018.

HOLM, M. Project-Based Instruction: A Review of the Literature on Effectiveness in Prekindergarten. River academic journal, v. 7, n. 2, p. 1-13, 2011.

LABOY-RUSH, D. Integrated STEM education through project-based learning. Learning.Com. 2011 Disponível em:

http://www.rondout.k12.ny.us/common/pages/DisplayFile.aspx. Acesso em: 17 de junho de 2017.

MEDEIROS, B. C; ARAÚJO, V. F; OLIVEIRA, M. K. A. Life Cycle Canvas (LCC): Um Modelo Visual para a Gestão do Ciclo de Vida do Projeto. Revista de Gestão e ProjetosGeP, v. 9, n. 1, p. 87-101, 2018.

MEDEIROS, B. C. et al. Planejando projetos com o Life Cycle Canvas (LCC): um estudo sobre um projeto de infraestrutura pública estadual. Exacta, v. 15, n. 1, p. 155-170, 2017.

MEDEIROS, B. C; SILVA, R. R. Gestão Visual em Projetos: Analisando os modelos de canvas à luz do Guia PMBOK®. In: Simpósio Internacional de Gestão de Projetos, Inovação e Sustentabilidade, 6., 2017, São Paulo. Anais. São Paulo: Uninove, 2017. p. 110 .

MEDEIROS, B. C; VERAS, M. Life Cycle Canvas: gestão dinâmica de projetos. Mundo Project Management, v. 12, n. 70, p. 76, 2016.

PAULO, M. C. C.; COSTA, D. M.; ANDRADE, R. M. M. Desafios da gestão universitária contemporânea: presenteísmo e seus impactos no desenvolvimento discente. Revista Gestão Universitária na América Latina - GUAL, Florianópolis, p. 01-19, 2018.

PAVANELO, E.; LIMA, R. Sala de Aula Invertida: a análise de uma experiência na disciplina de Cálculo I. Boletim de Educação Matemática, v. 31, n. 58, 2017.

PEREIRA, M. A. C.; BARRETO, M. A. M.; PAZETI, M. Application of Project-Based Learning in the first year of an Industrial Engineering Program: lessons learned and challenges. Production, v. 27, n. SPE, 2017. 
PMI, PMBOK GUIDE. Um guia do conhecimento em gerenciamento de projetos. Pennsylvania: Project Management Institute, 5ed. 2017.

RUAS, R.; COMINI, G. M. Aprendizagem e desenvolvimento de competências: articulando teoria e prática em programas de pós-graduação em formação gerencial. Cad.EBAPE.BR, Rio de Janeiro, v. 5, n. spe, p. 01-14, jan. 2007

SHARMA, N. et al. How we flipped the medical classroom. Medical Teacher, v. 37, n. 4, p. 327-330, 2015.

STEENKAMP, L. P.; HAGEDORN-HANSEN, D.; OOSTHUIZEN, G. A. Visual management system to manage manufacturing resources. Procedia Manufacturing, v. 8, p. 455-462, 2017.

VERAS, M. Gestão Dinâmica de Projetos: Life Cycle Canvas. Rio de Janeiro: Brasport, 2016.

VERAS, M. Negócio Baseado em Projetos (NBP). Rio de Janeiro: Brasport, 2018.

WANG, F. H. An exploration of online behaviour engagement and achievement in flipped classroom supported by learning management system. Computers \& Education, v. 114, p. 79-91, 2017.

ZUBER, W. J. The flipped classroom, a review of the literature. Industrial and Commercial Training, v. 48, n. 2, p. 97-103, 2016. 